\title{
Zahl der Pflegebedürftigen steigt stärker als vorausgesagt
}

Die Zahl der Pflegebedürftigen in Deutschland wird in den nächsten Jahren deutlich stärker steigen als bisher erwartet. Schon im Jahr 2015 gab es rund 40.000 Pflegebedürftige mehr als bislang angenommen. Dies machte der Vorstandsvorsitzende der Barmer GEK, Dr. Christioph Straub, bei der Vorstellung des diesjährigen Barmer GEK Pflegereports in Berlin deutlich.

Die neuen Prognosen des Pflegereports beruhen erstmals auf dem Zensus 2011 des Statistischen Bundesamtes, in dem festgestellt wurde, dass die deutsche Bevölkerung um etwa 1,5 Millionen Menschen kleiner ist als bis dahin angenommen. Auf der Basis dieser Zahlen wurden die Modellrechnungen der Krankenkasse bis zum Jahr 2060 nun angepasst.

2060 werden laut Pflegereport rund 4,52 Millionen Menschen in Deutschland pflegebedürftig sein - etwa 221.000 mehr als bisher angenommen. „In früheren Berechnungen wurde der Anteil älterer Menschen an der Bevölkerung unterschätzt“, sagte Straub. Steigen wird demnach vor allem die Zahl der Hochbetagten in der Bevölkerung, also derjenigen, die älter als 90 Jahre sind und dementsprechend häufiger auf Hilfe angewiesen sind.

Nur eine Woche vor der Vorstellung des Pflegereports hatte der Bundestag die seit langem erwartete Pflegereform beschlos- sen, die am 1. Januar 2017 in Kraft treten soll. Wichtigste Neuerung darin: In Zukunft wird es statt der bisherigen drei Pflegestufen fünf Pflegegrade geben. Dies ermögliche eine bessere Einstufung der Pflegebedürftigen: Rund 500.000 Menschen würden dann zusätzliche Leistungen bekommen, so rechnet das Bundesgesundheitsministerium. Vor allem für Demenzkranke steigen die Pflegesätze zukünftig deutlich an. Pflege-Experte Heinz Rothgang, der auch den Barmer GEK Pflegereport verantwortet, bezeichnete die beschlossene Reform als „ausgesprochen großzügig“.

Das bisherige System der sogenannten Minutenpflege wird mit der Reform abgelöst. Für Pflegekräfte ändere sich jedoch der Zeitdruck für die Pflege nicht, sagte Rothgang. In den im vorangegangenen Pflegereport stark in den Fokus geratenen Bereich der zahnärztlichen Versorgung in Pflegeheimen sei allerdings seit dem vergangenen Jahr Bewegung gekommen, sagte Rothgang dem DFZ. Es gebe eine Vielzahl von Projekten und Initiativen zur Verbesserung der zahnärztlichen Betreuung von Pflegebedürftigen. Auch wenn der Pflegereport das Thema in diesem Jahr nicht mehr aufgegriffen habe, sei es nicht in Vergessenheit geraten. „Da ist viel im Gange, um die Finanzierung dafür wird derzeit noch verhandelt“, sagte Rothgang.

\section{Statistik der Bundeszahnärztekammer}

\section{Zahnärzteschaft in Deutschland wird immer älter}

Deutschlands Zahnärzteschaft wächst kontinuierlich. Entgegen aller Unkenrufe, dass der Zahnarztberuf immer unattraktiver wird, steigt die Zahl der Zahnärzte seit Jahren stetig an: Gab es zur Jahrtausendwende im gesamten Bundesgebiet noch 78.742 Zahnärzte, so waren es im vergangenen Jahr mehr als 91.000. Dies geht aus dem aktuellen Statistischen Jahrbuch der Bundeszahnärztekammer hervor. Gut 70.000 Zahnärzte davon sind demnach tatsächlich als Zahnärzte tätig, rund 53.000 niedergelassen in eigener Praxis.

Um gut das Doppelte angestiegen ist in den vergangenen zehn Jahren die Zahl der angestellten Zahnärzte und Zahnärztinnen auf mehr als 14.000. Etwa 3000 Zahnärzte arbeiten zwar in ihrem Beruf, aber nicht in einer Zahnarztpraxis, und immerhin 20.000 Kolleginnen und Kollegen, haben den Zahnarztkittel an den Haken gehängt und gehen einer anderen Tätigkeit nach.

Bemerkenswert an der Zahlenschau ist der stete Zuwachs des Frauenanteils in der Zahnärzteschaft. Während es bei den männlichen Kollegen gerade einmal 1000 Zahnärzte mehr gibt als vor zehn Jahren, sind es bei den Frauen fast 8000 mehr. 2014 waren von den gut 91.000 Zahnärzten in Deutschland rund
40.000 Frauen - von denen drei Viertel auch als Zahnärztin tätig waren. Der Frauenanteil unter den zahnärztlich tätigen Zahnärzten stieg im neuen Jahrtausend besonders augenfällig in den alten Bundesländern auf mehr als 40 Prozent, während er in den neuen Bundesländern weitgehend konstant auf knapp unter 60 Prozent blieb.

Deutlich wird in der Statistik allerdings auch, dass die deutsche Zahnärzteschaft immer älter wird. Der „Durchschnittszahnarzt“ ist 48,3 Jahre alt - wobei die männlichen Kollegen durchschnittlich etwas älter als 50 sind, Zahnärztinnen dagegen den Schnitt senken und auf knapp 45 Jahre kommen. Waren vor fünf Jahren noch fünf Prozent aller Zahnärzte älter als 65 Jahre, sind es inzwischen schon acht Prozent - fast 80 Prozent davon sind Männer. Eine Zahl, die sich in den nächsten Jahrzehnten relativieren dürfte: Während an den Universitäten fast gleichbleibend viele junge Zahnmediziner (rund 13.000) ausgebildet werden, steigt der Frauenanteil kontinuierlich - im Jahr 2014 waren rund 65 Prozent aller Studierenden der Zahnmedizin weiblich. 